Diabetologe 2009 · 5:513-513

DOI 10.1007/s11428-009-0495-9

(c) Springer Medizin Verlag 2009

\author{
H. Finck \\ Vorsitzender des Ausschusses Soziales der DDG, Hünfeld
}

\title{
Sozialmedizinische Aspekte des Diabetes mellitus
}

und den Diabetesteams Hilfe und Unterstützung bei der Bewältigung von sozialen Problemen und eine kompetente Beratung bei folgenden sozialmedizinischen Themen:

- Diabetes und Fahrtauglichkeit/Fahreignung,

- Diabetes und Schwerbehinderung,

- Diabetes und Beruf,

- Diabetes bei Kindern und Jugendlichen, sozialpädiatrische Aspekte.

Unter Berücksichtigung der Tatsache, dass die hier dargestellten Problemfelder im sozialen, sozialmedizinischen, sozialrechtlichen, arbeitsmedizinischen und arbeitsrechtlichen Bereich sehr häufig mit Rechtsfolgen für alle Beteiligten einhergehen, muss deutlich darauf hingewiesen und aufmerksam gemacht werden, dass die rechtsrelevanten Themen immer auch in Patientenschulungsprogramme sowie insbesondere auch in individuelle Schulungen mit aufgenommen werden sollten, zumal im Versicherungs- und Schadensfall seitens der Rechtsinstanz immer hinterfragt werden wird, ob dem Schadensoder Unfallverursacher die Tauglichkeitsoder Eignungs- oder Funktionseinschränkungen sowie auch die Kompensationsmöglichkeiten bekannt waren.

Die Aufklärung über etwaige Tauglichkeits-, Eignungs- oder Funktionseinschränkungen sollte in der Patientenakte dokumentiert sein.

Diese Tatbestände sollen insbesondere den Diabetologen in Kliniken und in Schwerpunktpraxen sowie den Diabetesteams mit Diabetesberaterinnnen und assistentinnen wegen der Rechtsrelevanz und der Rechtsfolgen nochmals verdeutlicht werden.
Ich wünsche Ihnen mit diesem Themenheft eine informative Lektüre.

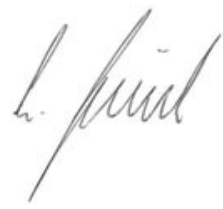

Hermann Finck

\section{Korrespondenzadresse}

Dr. H. Finck

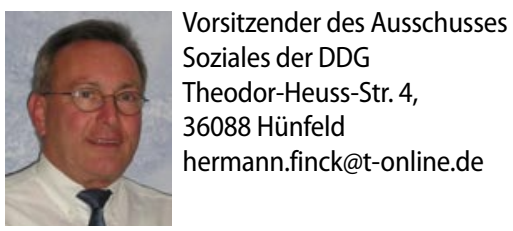

Der Patient mit Diabetes mellitus erwartet auch von den behandelnden Ärzten 